\title{
A recuperação intraoperatória de células sanguíneas é eficaz em cirurgias de quadril?*
}

\section{Is Intraoperative Blood Cell Salvage Effective in Hip Surgery?}

\author{
Nara Granja Nunes ${ }^{1}$ José Alberto Alves Oliveira ${ }^{20}$ Francisca Magna Prado Bezerra ${ }^{1}$ \\ Velma Dias do Nascimento ${ }^{3}$ Danielle Maia Holanda Dumaresq ${ }^{1}$ Manoel Cláudio Azevedo Patrocinio ${ }^{1}$ \\ ${ }^{1}$ Serviço de Anestesiologia, Instituto Dr. José Frota, Fortaleza, CE, \\ Brasil \\ ${ }^{2}$ Serviço de Ortopedia e Traumatologia, Hospital Infantil Albert Sabin, \\ Hospital Geral de Fortaleza, Fortaleza, CE, Brasil \\ ${ }^{3}$ Serviço de Hemoterapia, Instituto Dr. José Frota, Fortaleza, CE, Brasil

\begin{abstract}
Endereço para correspondência José Alberto Alves Oliveira, MD, Hospital Infantil Albert Sabin-Hospital Geral de Fortaleza, Serviço de Ortopedia e Traumatologia, Rua Bruno Porto, 600, apto 2201-A, Parque Iracema, Fortaleza, CE, 608-24010, Brasil

(e-mail: bertoalvesoliveira@gmail.com).
\end{abstract}

Rev Bras Ortop 2019;54:377-381.

\section{Resumo \\ Palavras-chave \\ - quadril/cirurgia \\ - fraturas do fêmur \\ - transfusão de sangue autóloga \\ - transfusão sanguínea \\ Objetivo O estudo visa avaliar a eficácia da recuperação intraoperatória de sangue (RIOS) na redução de hemotransfusão alogênica em pacientes submetidos à cirurgia por fratura de fêmur e quadril. \\ Métodos Coorte prospectiva com 38 pacientes submetidos a cirurgia traumatoló- gica para fraturas em quadril e transtrocantéricas de fêmur, divididos em dois grupos em um hospital de ensino de agosto de 2015 a fevereiro de 2017. Pacientes com qualquer enfermidade ou condição infecciosa foram excluídos do presente estudo. $\mathrm{O}$ grupo RIOS (19 pacientes) recebeu sangue autólogo com a utilização de Cell Saver, enquanto o grupo controle (19 pacientes) recebeu apenas sangue alogênico, quando necessário.. Grupos comparados em relação ao gênero, idade na cirurgia, escala da Sociedade Americana de Anestesiologistas (ASA) (I, II ou III), uso intraoperatório da RIOS, volume sanguíneo reinfundido pela RIOS, parâmetros hematimétricos pré- e pós- operatórios, volume intra e pós-operatório de sangue alogênico transfundido. Dados processados no software SPSS Statistics for Windows, Versão 20.0 (IBM Corp, Armonk, NY, EUA). \\ Resultados Sem diferenças significativas entre os grupos com as variáveis: idade, gênero e ASA. Percebeu-se que os valores finais de hemoglobina e hematócrito (no $1^{\circ}$ dia de pós-operatório) foram mais elevados no grupo que utilizou o dispositivo $(p<0,05)$. Não houve redução significativa da transfusão alogênica intra e pós- operatória no grupo RIOS em comparação ao controle. \\ Conclusões O presente estudo constatou que a RIOS não foi eficaz em reduzir a transfusão alogênica no intra e pós-operatório de pacientes submetidos à cirurgia de fêmur transtrocantérica e de quadril.}

* Trabalho feito no Instituto Dr. José Frota, Fortaleza, CE, Brasil. (D) José Alberto Alves Oliveira's ORCID is https://orcid.org/0000-00026424-1490.

recebido

21 de Janeiro de 2018 aceito

06 de Agosto de 2018
DOI https://doi.org/

10.1055/s-0039-1693054. ISSN 0102-3616.
Copyright $\odot 2019$ by Sociedade Brasileira License terms de Ortopedia e Traumatologia. Published by Thieme Revnter Publicações Ltda, Rio de Janeiro, Brazil 


\begin{abstract}
Keywords

- hip/surgery

- femoral fractures

- blood transfusion, autologous

- blood transfusion

Objective The present study aims to evaluate the efficacy of cell salvage (CS) as a method of reducing allogeneic hemotransfusion in patients submitted to surgeries for trochanteric and hip injuries.

Methods Prospective cohort with 38 patients submitted to hip or trochanteric surgeries divided into 2 groups in a school hospital from August 2015 to February 2017. Patients with any malignancy or infectious condition were excluded from the study. The CS group (19 patients) received autologous blood using Cell Saver, whereas the control group (19 patients) received just allogeneic blood, if needed. The comparative of the hematimetric parameters and blood transfusion of the patients were assessed both preoperatively and postoperatively, as clinical and surgical characteristics such as age, gender, American Society of Anesthesiologists (ASA, na sigla em inglês) scale and type of surgery. Data was processed in SPSS Statistics for Windows, Versions 20.0 (IBM Corp, Armonk, NY, USA).

Results There were no differences regarding the clinical parameters studied (age, gender, and ASA). The hematimetric parameters on the $1^{\text {st }}$ postoperative day were higher in the CS group $(p<0.05)$. No significant reduction of intraoperative and postoperative allogeneic transfusion was found.

Conclusion This study found that CS was not effective in reducing allogeneic transfusion in the intraoperative and postoperative period of patients submitted to trochanteric and hip surgery.
\end{abstract}

\section{Introdução}

Uma das principais causas de morbidade em cirurgias como a artroplastia total do quadril (ATQ) e de fratura transtrocanteriana de fêmur é a elevada perda sanguínea, ${ }^{1-3}$ a qual, segundo alguns estudos, pode exceder $500 \mathrm{ml}$ no intraoperatório e $750 \mathrm{ml}$ no pós-operatório. ${ }^{4}$

O sangue homólogo é frio, acidótico, rico em potássio, com baixas taxas de 2,3-DPG, sem conseguir transportar oxigênio adequadamente por até 24 horas. ${ }^{5}$ Carrega, assim, o risco de efeitos adversos imunológicos e não imunológicos, tais como uma maior taxa de infecção pós-operatória, risco de contaminação, transmissão de doenças (CMV, HIV, hepatites), reações de hipersensibilidade, hemólise intravascular, coagulopatia induzida por transfusão, insuficiência renal, desenvolvimento de autoanticorpos com dificuldade de nova compatibilidade posteriormente, aumento do tempo de internação hospitalar e o aumento da mortalidade. ${ }^{1,5-7}$

Com o intuito de diminuir a necessidade deste tipo de transfusão em cirurgias com grande perda sanguínea esperada, diversas técnicas de gerenciamento de sangue são utilizadas: doação de sangue autólogo no pré-operatório, hemodiluição normovolêmica, anestesia hipotensiva, ácido aminocapróico, ácido tranexâmico, eritropoietina pré-operatória e sistema de resgate de células sanguíneas com sua reinfusão no intraoperatório. ${ }^{8,9}$

A recuperação intraoperatória de sangue (RIOS) é um sistema de resgate de células intraoperatório que coleta o sangue do campo operatório por aspiração, lava e filtra os resíduos, tais como restos celulares e bioquímicos. Após o processo de filtração, os eritrócitos do paciente retornam para ele. $^{10}$
Apesar das vantagens deste método, não está claro se sua utilização reduz a necessidade de hemotransfusões alogênicas nas cirurgias de quadril e fêmur proximal.

O presente estudo visa avaliar a eficácia do Cell Saver como método de redução da hemotransfusão alogênica em pacientes submetidos a cirurgias eletivas de fêmur proximal e quadril pós-trauma.

\section{Materiais e Métodos}

Trata-se de um estudo do tipo coorte prospectiva com uma amostra composta por 38 pacientes submetidos a cirurgia para fratura de fêmur transtrocantérica ou de quadril, no serviço de traumatologia e ortopedia da nossa instituição, entre agosto de 2015 e fevereiro de 2017.

Foi utilizado apenas um sistema de recuperação intraoperatório de sangue no período estudado, o Medtronic Autolog, (Medtronic inc. Minneapolis, USA). O dispositivo foi utilizado em 19 pacientes (grupo RIOS) e não foi utilizado em nos outros 19 (grupo controle). Determinou-se o número da amostra por estudos prévios semelhantes encontrados na literatura. $1,7,11,12$

Os pacientes que apresentaram no pré-operatório histórico de distúrbios da hemostasia, baixa contagem de plaquetas $(<100.000)$, alterações no tempo de atividade da protrombina (TAP) / razão normalizada internacional (INR), tempo de tromboplastina parcialmente ativada (TTPA); eventos tromboembólicos anteriores à cirurgia, ou histórico familiar de tromboembolismo; escala da Sociedade Americana de Anestesiologistas (ASA, na sigla em inglês) $>3$, aqueles que por crenças religiosas (Testemunha de Jeová) não permitem transfusão de sangue alogênico, 
portadores de neoplasias e/ou de quadro infeccioso sistêmico foram excluídos da amostra.

Em nenhum paciente foi utilizado sangue autólogo prédoado, hemodiluição isovolumétrica no intraoperatório, nem eritropoetina (EPO) durante o pré- e pós-operatório. Na indução anestésica, dentre os medicamentos antifibrinolíticos, ácido aminocapróico (dose de $4 \mathrm{~g}$ ) foi utilizado em 3 pacientes no grupo RIOS e em um paciente no grupo controle, e ácido tranexâmico (dose de $1 \mathrm{~g}$ ) em outro 4 pacientes de cada grupo.

Os pacientes foram colocados em decúbito lateral na mesa de cirurgia para o acesso de Kocher Langenbeck nas cirurgias de quadril, enquanto nas cirurgias de fêmur proximal, os pacientes foram colocados em decúbito dorsal. Nas artroplastias totais do quadril, foi utilizada a conformação do fêmur proximal $(A, B, C)$ e o índice cortical descritos por Dorr et al apud Semkiw et $\mathrm{al}^{11}$ para cimentação ou não do componente femoral. Foi realizada anestesia com bloqueio de neuroeixo (raquianestesia ou peridural) com volume estabelecido pelo anestesista conforme a necessidade cirúrgica, associado ou não a anestesia geral.

Os parâmetros para a transfusão sanguínea incluiu sinais e sintomas de anemia: redução do volume urinário $<30 \mathrm{ml} / \mathrm{h}$ aferido pela sonda vesical, taquicardia ( $>100$ batimentos/ minunto) e hipotensão $(<100 \mathrm{~mm} \mathrm{Hg}$ de pressão arterial sistólica) refratária à expansão volêmica e um nível de hemoglobina $<8 \mathrm{~g} / \mathrm{dl}$ pela gasometria associado a sinais ou sintomas de anemia, ou um nível de hemoglobina $<7$ $\mathrm{g} / \mathrm{dl}$ independente de sinais ou sintomas de anemia. A decisão de hemotransfusão alogênica durante a cirurgia foi uma decisão conjunta entre o cirurgião e o anestesista.

No período pós-operatório, os pacientes permaneciam em unidade de recuperação pós-anestésica em torno de 2 horas pós-operatórias. Neste período, os mesmos parâmetros para transfusão sanguínea foram adotados, sendo a decisão de transfundir o paciente tomada de forma conjunta entre o cirurgião e o anestesista. Após este período, o paciente recebia alta para a enfermaria, ficando a decisão de transfundir a cargo do cirurgião responsável. Vale salientar que todas as feridas operatórias foram fechadas sem a utilização de drenos de sucção.

Os grupos foram comparados de acordo com os seguintes dados dos prontuários médicos: gênero; idade na cirurgia; escala ASA (I, II ou III); uso intraoperatório da RIOS; volume sanguíneo reinfundido pela RIOS; níveis pré- e pós-operatórios de hemoglobina ( $\mathrm{Hb}$ ) e de hematócritos $(\mathrm{Ht})$; volume de hemácias intra e pós-operatório de sangue alogênico transfundido.

Os dados foram processados no software SPSS Statistics for Windows, Versão 20.0 (IBM Corp, Armonk, NY, EUA), licença ${ }^{\circ}$ 10101131007, quando foram calculadas as medidas: média e desvio padrão (DP). As comparações das médias, segundo as características gerais, entre os grupos RIOS e controle, dos níveis hematimétricos ( $\mathrm{Hb}$ e $\mathrm{Ht}$ ) e da necessidade de transfusão sanguínea (intra e pós-operatória) foram feitas pelos testes t de Student. Foi fixado o nível de confiança de 0,05.

Conforme a Resolução 466/2012 do Conselho Nacional de Saúde, o presente estudo foi aprovado pelo Comitê de Ética em Pesquisa da referida instituição com o parecer 1.702.480, e todos os participantes assinaram o termo de consentimento livre esclarecido.

\section{Resultados}

Uma análise descritiva foi realizada para resumir as características dos pacientes e dos procedimentos cirúrgicos. Um total de 19 (50\%) pacientes foram operados utilizando o sistema de resgate de células intraoperatório, e em 19 (50\%) o sistema não foi utilizado, constituindo o grupo controle ( - Tabela $\mathbf{1}$ ).

A idade média dos pacientes foi de $58,21 \pm 20,03$ anos no grupo controle e de $46,89 \pm 18,92$ anos no grupo RIOS $(p>0,05)$. Não houve diferenças significativas entre os grupos com as demais variáveis (gênero, ASA I/II/III). A média de volume sanguíneo recuperado no intraoperatório e reinfundido ao paciente foi de $335,47 \pm 255,86 \mathrm{ml}$ no grupo que utilizou o dispositivo (- Tabela 1 ).

Ao se avaliar os níveis hematimétricos dos pacientes entre os dois grupos, percebeu-se que os valores finais de $\mathrm{Hb}$ e de $\mathrm{Ht}$ (no $1^{\circ}$ dia de pós-operatório) foram mais elevados no grupo que utilizou a RIOS $(p<0,05)$ ( - Tabela 2 ).

Ao se comparar o volume de hemácias alogênicas transfundido entre os dois grupos, percebeu-se que não houve diferença no intraoperatório ( $\mathrm{p}>0,05)$. Já no período pósoperatório, houve uma menor quantidade de transfusão nos pacientes que fizeram uso da RIOS, porém tal diferença não se mostrou significativa $(p>0,05)$. Quando se considerou a soma dos períodos (intra e pós-operatório), não se evidenciou uma redução no volume total de hemoconcentrados alogênicos transfundidos nos pacientes que utilizaram o dispositivo em comparação com o grupo controle $(\mathrm{p}>0,05)(-$ Tabela 3$)$.

\section{Discussão}

Apesar da triagem das bolsas de sangue ter melhorado consideravelmente a segurança nos últimos anos, ainda existem riscos conhecidos, como potenciais reações transfusionais e de aloimunização, bem como associados ao risco de contrair doenças infecciosas: vírus da imunodeficiência humana (HIV) 1:1.930.000, hepatite B 1:137.000, hepatite C $1: 1.000 .000$ e sepse bacteriana. ${ }^{9}$

Técnicas de coleta de sangue autólogo são fonte de discussão desde sua introdução há mais de 30 anos. ${ }^{13}$ Ainda há dúvidas, porém, sobre a eficiência e os custos relacionados a estas técnicas em algumas cirurgias e a discriminação de seu uso. ${ }^{13,14}$

Indicações para o uso da RIOS incluem: antecipação de perda sanguínea $>1000 \mathrm{~mL}$, média de uma ou mais unidade transfusional de sangue alogênico no pós-operatório, a recusa transfusional por motivos religiosos, uma baixa hemoglobina pré-operatória, fatores de risco para sangramento, ou se $>10 \%$ dos pacientes requerem transfusão para aquele tipo de cirurgia. ${ }^{13}$

As contraindicações absolutas para utilização desse dispositivo são: situações de hemólise (sangue misturado com 
380 A eficácia da recuperação intraoperatória de sangue Nunes et al.

Tabela 1 Comparação de características clínicas e cirúrgicas entre os dois grupos

\begin{tabular}{|c|c|c|c|}
\hline & $\begin{array}{l}\text { Controle } \\
(n=19 / 50 \%)\end{array}$ & $\begin{array}{l}\text { RIOS } \\
(n=19 / 50 \%)\end{array}$ & valor-p \\
\hline Idade (anos) & $58,21 \pm 20,03$ & $46,89 \pm 18,92$ & $>0,05$ \\
\hline \multicolumn{3}{|l|}{ Sexo } & $>0,05$ \\
\hline Feminino & $6(60,00 \%)$ & $4(40,00 \%)$ & \\
\hline Masculino & $13(46,42 \%)$ & $15(53,58 \%)$ & \\
\hline \multicolumn{3}{|l|}{ Asa } & $>0,05$ \\
\hline 1 & $8(50 \%)$ & $8(50 \%)$ & \\
\hline II & $6(46,15 \%)$ & $7(53,85 \%)$ & \\
\hline III & $5(55,55 \%)$ & $4(44,45 \%)$ & \\
\hline Volume recuperado no intraoperatório $(\mathrm{mL})$ & - & $335,47 \pm 255,86$ & \\
\hline \multicolumn{4}{|l|}{ Cirurgias } \\
\hline Osteossíntese de fêmur & $7(36,84 \%)$ & $1(5,26 \%)$ & \\
\hline Osteossíntese de acetábulo & $4(21,05 \%)$ & $5(26,32 \%)$ & \\
\hline Artroplastia total do quadril & $6(31,58 \%)$ & $10(52,63 \%)$ & \\
\hline Artroplastia parcial do quadril (bipolar) & $2(10,53 \%)$ & - & \\
\hline Revisão de artroplastia do quadril & - & $3(15,79 \%)$ & \\
\hline
\end{tabular}

Tabela 2 Comparação dos valores de hemoglobina e de hematócritos no pré- e pós-operatório entre os dois grupos

\begin{tabular}{|l|l|l|l|}
\hline \multicolumn{2}{|l|}{ Controle $(\boldsymbol{n}=\mathbf{1 9 / 5 0 \% )}$} & RIOS $(\boldsymbol{n}=\mathbf{1 9 / 5 0 \% )}$ & valor-p $^{*}$ \\
\hline Hemoglobina(g/dL) & $11,17 \pm 1,33$ & $12,21 \pm 1,98$ & $\mathrm{p}>0,05$ \\
\hline Pré-operatória & $8,20 \pm 1,40$ & $9,78 \pm 1,74$ & $p=0,004$ \\
\hline $1^{\circ}$ Pós-operatório & $33,95 \pm 4,60$ & $37,07 \pm 5,84$ & $\mathrm{p}>0,05$ \\
\hline Hematócrito (\%) & $29,90 \pm 5,05$ & $p=0,004$ \\
\hline Pré-operatória & $25,36 \pm 4,08$ & \\
\hline $1^{\circ}$ Pós-operatório
\end{tabular}

P* pelo teste T de Student.

água, álcool ou peróxido de hidrogênio), anormalidades dos eritrócitos (por exemplo, anemia falciforme) ou procedimentos com contaminação fecal ou urinária. Outras relatadas são: malignidade, presença de partículas muito pequenas para serem filtradas e infecções sistêmicas, sob risco de disseminação dessas patologias. ${ }^{4,13}$

Em geral, os resultados do presente estudo não revelaram uma redução significativa no volume total transfundido no grupo que utilizou o aparelho. A média de volume recuperado foi de $335 \mathrm{~mL}$, compatível com autores como Leigheb et al, $^{4}$ Hawi et al ${ }^{15}$ e Buget et al, ${ }^{16}$ que também mostraram volumes sanguíneos transfundidos no perioperatório nos dois grupos semelhantes ao nosso ensaio (grupo RIOS: média $170,14 \mathrm{~mL}$; grupo controle: média $92,53 \mathrm{~mL}$ ), porém tal diferença entre os grupo foi significante, talvez pela maior amostra (143 pacientes).

A RIOS é um procedimento complexo, que requer uma equipe qualificada, tanto para o manuseio da máquina, quanto habilidade em aspiração do sangramento, além de ter como desvantagem o fato de não estar sempre

Tabela 3 Comparação entre a transfusão de concentrado de hemácias alogênicas entre os dois grupos

\begin{tabular}{|c|c|c|c|}
\hline & Controle $(n=19 / 50 \%)$ & $\operatorname{RIOS}(n=19 / 50 \%)$ & valor-p* \\
\hline \multicolumn{4}{|l|}{ Transfusão (mL) } \\
\hline Intraoperatória & $17,31 \pm 75,47$ & $23,94 \pm 73,38$ & $p=0,78$ \\
\hline Pós-operatória & $153,94 \pm 275,13$ & $67,00 \pm 163,16$ & $p=0,24$ \\
\hline Total & $171,26 \pm 275,26$ & $90,94 \pm 169,17$ & $p=0,28$ \\
\hline
\end{tabular}

$\mathrm{P}^{*}$ pelo teste T de Student. 
disponível. ${ }^{4}$ Segundo Herd et al, ${ }^{13}$ o uso do aparelho reduziu as taxas de transfusões perioperatórias com o decorrer dos anos no mesmo serviço hospitalar, atribuído ao aumento da competência e experiência com a máquina, recuperando um maior volume sanguíneo.

Há vários tipos de cirurgia em fêmur e quadril, que levam a diferentes volumes de perda sanguínea. ${ }^{13} \mathrm{O}$ risco de perda sanguínea perioperatória aumenta com o grau de dificuldade do procedimento, especialmente em casos de revisão. ${ }^{15}$ No nosso estudo, apesar de seleção aleatória dos grupos, o grupo RIOS apresentou um maior número de cirurgias de revisão de artroplastia de quadril (reconhecidas como maior possibilidade de sangramento).

No presente estudo, não houve relatos de complicações como embolia aérea, coagulopatia dilucional, bacteremia, hipervolemia, overdose de anticoagulante e hemoglobinúria.

Alguns dados não foram registrados, como qual componente acetabular foi usado, se era artroplastia em estágio único ou não, cimentada ou não, reduzindo a eficácia de comprovação da homogeneidade dos procedimentos cirúrgicos. Isto pode nos levar a indagar se o grupo RIOS foi utilizado para cirurgias mais complexas, com sangramento intraoperatório significativamente mais elevado, apresentando, portanto, um fator de confusão para os resultados, assim como no estudo de Garvin et al. ${ }^{17}$ Outra variável utilizada em nosso estudo que pode ter afetado os resultados foi a utilização de agentes antifibrinolíticos nos dois grupos.

\section{Conclusão}

O presente estudo constatou que a recuperação intraoperatória de sangue não foi eficaz em reduzir a transfusão de sangue alogênico no intraoperatório, no pós-operatório e no período total em pacientes submetidos a cirurgias de fêmur proximal e de quadril por trauma. No entanto, apesar de não diminuir o número de transfusões, a média dos valores de $\mathrm{Hb}$ e Ht no $1^{\circ}$ pós-operatório foi melhor no grupo que utilizou a RIOS. Talvez um trabalho com mais pacientes pudesse chegar a valores significativos, conforme a literatura.

O presente estudo não avaliou custos operacionais nem tempo de internação hospitalar. Os autores acreditam que são necessários mais estudos prospectivos, randomizados, que avaliem não só a eficácia do Cell Saver, mas também seu custo-efetividade.

Conflitos de Interesses

Os autores declaram não haver conflitos de interesses.

\section{Referências}

1 Elawad AA, Öhlin AK, Berntorp E, Nilsson IM, Fredin H. Intraoperative autotransfusion in primary hip arthroplasty. A randomized comparison with homologous blood. Acta Orthop Scand 1991;62 (06):557-562

2 Bridgens JP, Evans CR, Dobson PM, Hamer AJ. Intraoperative red blood-cell salvage in revision hip surgery. A case-matched study. J Bone Joint Surg Am 2007;89(02):270-275

3 McMurray MR, Birnbaum MA, Walter NE. Intraoperative autologous transfusion in primary and revision total hip arthroplasty. J Arthroplasty 1990;5(01):61-65

4 Leigheb M, Pogliacomi F, Bosetti M, Boccafoschi F, Sabbatini M, Cannas $M$, et al. Postoperative blood salvage versus allogeneic blood transfusion in total knee and hip arthroplasty: a literature review. Acta Biomed 2016;87(Suppl 1):6-14

5 Catling S, Joels L. Cell salvage in obstetrics: the time has come. BJOG 2005;112(02):131-132

6 Kumar A. Perioperative management of anemia: limits of blood transfusion and alternatives to it. Cleve Clin J Med 2009;76(04, Suppl 4):S112-S118

7 Phillips SJ, Chavan R, Porter ML, Kay PR, Hodgkinson JP, Purbach B, et al. Does salvage and tranexamic acid reduce the need for blood transfusion in revision hip surgery? J Bone Joint Surg Br 2006;88 (09):1141-1142

8 Oliveira JAA, Façanha Filho FAM, Fernandes FV, Almeida PC, de Oliveira VF, Lima Verde SR. Is cell salvage cost-effective in posterior arthrodesis for adolescent idiopathic scoliosis in the public health system? J Spine Surg 2017;3(01):2-8

9 Lemaire R. Strategies for blood management in orthopaedic and trauma surgery. J Bone Joint Surg Br 2008;90(09):1128-1136

10 Oliveira JAA, Façanha Filho FAM, Feijão SX, Fernandes FV, Almeida PC, Carlos LM, et al. Influence of Intraoperative Blood Salvage on Surgery for Scoliosis. Coluna/Columna 2017;16(01):33-37

11 Semkiw LB, Schurman DJ, Goodman SB, Woolson ST. Postoperative blood salvage using the Cell Saver after total joint arthroplasty. J Bone Joint Surg Am 1989;71(06):823-827

12 Shulman G, Grecula MJ, Hadjipavlou AG. Intraoperative autotransfusion in hip arthroplasty. Clin Orthop Relat Res 2002; (396):119-130

13 Herd JM, Joseph JJ, McGarvey M, Tsimbouri P, Bennett A, Meek RM, et al. Intraoperative cell salvage in revision hip surgery. Ann Med Surg (Lond) 2014;3(01):8-12

14 van Bodegom-Vos L, Voorn VM, So-Osman C, Vliet Vlieland TP, Dahan A, Koopman-van Gemert AW, et al. Cell Salvage in Hip and Knee Arthroplasty: A Meta-Analysis of Randomized Controlled Trials. J Bone Joint Surg Am 2015;97(12):1012-1021

15 Hawi N, Kendoff DO, Hessling U, Haasper C, Gehrke T, Citak M. Effectiveness of an autologous transfusion system following cemented and non-cemented revisions of total hip arthroplasty. Int Orthop 2014;38(08):1603-1608

16 Buget MI, Dikici F, Edipoğlu IS, Yıldız E, Valiyev N, Kucukay S. Twoyear experience with cell salvage in total hip arthroplasty. Braz J Anesthesiol 2016;66(03):276-282

17 Garvin KL, Feschuk CA, Sekundiak TD, Lyden ER. Blood salvage and allogenic transfusion needs in revision hip arthroplasty. Clin Orthop Relat Res 2005;441(441):205-209 